\title{
Caracterización del crecimiento y producción de rodales forestales a partir de datos PNOA-LiDAR multitemporales en la provincia de Lugo
}

Characterization of the growth and production of forest stands from multi-temporal PNOA-LiDAR data in the province of Lugo

\author{
Salgado, L. ${ }^{1,2 *}$; Colina, A. ${ }^{2}$; Docampo, M.L. ${ }^{1}$; López-Sánchez, C.A. ${ }^{3}$ \\ 'Departamento de Ingeniería Agroforestal, Escola Politécnica Superior de Enxeñaría, Campus de Lugo, \\ Universidade de Santiago de Compostela, España. \\ ${ }^{2}$ Instituto de Recursos Naturales y Ordenación del Territorio, Campus de Mieres, \\ Universidad de Oviedo, España. \\ ${ }^{3}$ Departamento de Biología de Organismos y Sistemas, Escuela Politécnica de Mieres, \\ Campus de Mieres, Universidad de Oviedo, España
}




\title{
Resumen
}

El cálculo de volúmenes forestales es una de las aplicaciones más comunes de la teledetección para apoyar la gestión sostenible de los ecosistemas forestales. En este estudio, se utilizan datos del $4^{\circ}$ Inventario Forestal Nacional (IFN-4) y de la $1^{\mathrm{a}}$ y $2^{\mathrm{a}}$ encuesta nacional de Exploración Láser Aerotransportada (ALS) para desarrollar modelos de rendimiento predictivo y estimaciones directas para las tres principales especies forestales comerciales (Eucalyptus globulus, Pinus pinaster y Pinus radiata) cultivadas en la provincia de Lugo. La integración de ambos tipos de datos requirió una armonización previa debido a las diferencias en el tiempo de adquisición de los datos y las dificultades para geolocalizar con precisión las parcelas del IFN-4.

Los datos armonizados de E. globulus, $P$. pinaster y $P$. radiata se han utilizado para desarrollar modelos predictivos para determinar el volumen total con corteza (VCC) en un punto t diferente del de captura de los datos PNOA-LiDAR. Se ha calculado el incremento anual de volumen con corteza (IAVC) mediante la relación entre las variables de las parcelas IFN-4, con las métricas derivadas de los datos de PNOA-LiDAR, mediante el método de regresión lineal Random Forest (RF) y varias técnicas de aprendizaje automático. Estos datos también se utilizan para obtener valores VCC de forma directa mediante datos PNOA-LiDAR multitemporales, siendo estos últimos capturados en el tiempo t de proyección del otro método.

Palabras clave: Incremento anual de volumen con corteza, Inventario Forestal Nacional, Random Forest, volumen total con corteza.

\begin{abstract}
In this study, data from the 4th National Forest Inventory (IFN-4) and from the 1st and 2nd National Airborne Laser Exploration Survey (ALS) are used to develop predictive performance models and direct estimates for the three main commercial forest species (Eucalyptus globulus, Pinus pinaster and Pinus radiata) grown in the province of Lugo. The integration of both types of data required prior harmonization due to the differences in the time of data acquisition and the difficulties to accurately geolocate the IFN4 plots.

The harmonized data of E. globulus, $P$. pinaster and $P$. radiata have been used to develop predictive models to determine the total volume with cortex (VCC) at a point $t$ different from the capture of the PNOA-LiDAR data. The annual increase in volume with cortex (IAVC) was calculated by means of the relationship between the variables of the IFN4 plots, with the metrics derived from the PNOA-LiDAR data, by the Random Forest (RF) linear regression method and several techniques of machine learning. These data are also used to obtain VCC values directly using multi-temporal PNOA-LiDAR data, the latter being captured at the projection time $t$ of the other method.
\end{abstract}

Keywords: Annual increase total volume, National Forest Inventory, Random Forest, Total volume with cortex. 


\section{Introducción}

Dentro del sector forestal, la información de los recursos madereros y su variación temporal tiene importancia medioambiental y económica básica para propietarios, empresas, administradores forestales e investigadores (Novo-Fernández et al., 2019), siendo el inventario forestal basado en la medición en campo del diámetro a la altura del pecho (dbh) y la altura total (h) de los árboles en numerosas parcelas el método más usado para obtener esta información (Nilson et al., 2016), a pesar de caracterizarse por ser un proceso arduo y costoso (Alberti et al., 2016). Además, este método el usado en la elaboración del Inventario Forestal Nacional (IFN) base de datos pública forestal utilizada para tareas de gestión forestal.

A principios del siglo XXI se produce una revolución en el sector forestal con la aparición de sensores remotos activos, que a través de la tecnología láser son una gran fuente de datos forestales gracias a su capacidad de recogida de datos espaciales georreferencidados de forma automática y eficiente (Wehr and Lohr, 1999). Destaca la tecnología LiDAR (Light Detection and Ranging), que irrumpe en el mercado reduciendo los costes y tiempos en la realización de inventarios forestales y en las estimaciones de biomasa a través de la modelización de variables dasométricas, y experimentando recientemente un gran desarrollo, debido a su entrada en el mercado comercial y el desarrollo software específico (Renslow, 2012).

Hoy en día los datos LiDAR están disponibles en múltiples adquisiciones temporales (Marinelli et al., 2018; McRoberts et al., 2015; Simonson et al., 2016; Zhao et al., 2018), abriendo la posibilidad para cuantificar dinámicas forestales (Dalponte et al., 2019), siguiendo estos estudios dos enfoques diferenciados. El primero de ellos predice los cambios de reservas de carbono/bioma$\mathrm{sa} /$ volumen forestal directamente a partir de métricas LiDAR multitemporales (Hopkinson et al., 2008; Englhart et al., 2013; Réjou-Méchain et al., 2015; Cao et al., 2016; Zhao et al., 2018), mientras que el segundo se basa en predecir las reservas de carbono/biomasa/volumen en cada instante temporal (Bollandsås et al., 2013; McRoberts et al., 2015; Réjou-Méchain et al., 2015; Bollandsås et al., 2017).

Con la unión de los datos del IFN con la información PNOA-LiDAR se produce un aumento sustancial de la información forestal (Jiménez et al., 2017). Además, con la publicación de la $2^{\mathrm{a}}$ cobertura PNOA-LiDAR, se abren nuevas líneas de investigación, pudiéndose obtener información de evolución forestal de forma directa con métricas LiDAR multitemporales.

El objetivo general de este trabajo es el estudio del crecimiento y producción de masas forestales a partir de datos PNOA-LiDAR multitemporales en la provincia de Lugo, analizando los datos de las parcelas del Cuarto Inventario Forestal Nacional (IFN-4), realizando un ajuste de modelos predictivos para la estimación del Volumen con corteza (VCC) e incremento anual en volumen con corteza (IAVC), a partir de datos del IFN-4 y la $1^{\text {a }}$ cobertura PNOA-LiDAR, estimación del VCC en dos instantes temporales ( $1^{\mathrm{a}}$ y $2^{\mathrm{a}}$ cobertura PNOA-LiDAR) 
y aplicación del modelo IAVC a la $1^{\text {a }}$ cobertura PNOA-LiDAR, validación de la estimación del VCC correspondiente a la $2^{\mathrm{a}}$ cobertura PNOA-LiDAR calculado respecto al valor obtenido por proyección del VCC de la $1^{\mathrm{a}}$ cobertura PNOALiDAR a la fecha de la $2^{\mathrm{a}}$ cobertura PNOA-LiDAR mediante el modelo IAVC.

\section{Material y Métodos}

\subsection{Zona de estudio}

El área de estudio se ubica en el noroeste España, en la comunidad autónoma de Galicia, abarcando toda la provincia de Lugo (Fig. 1).

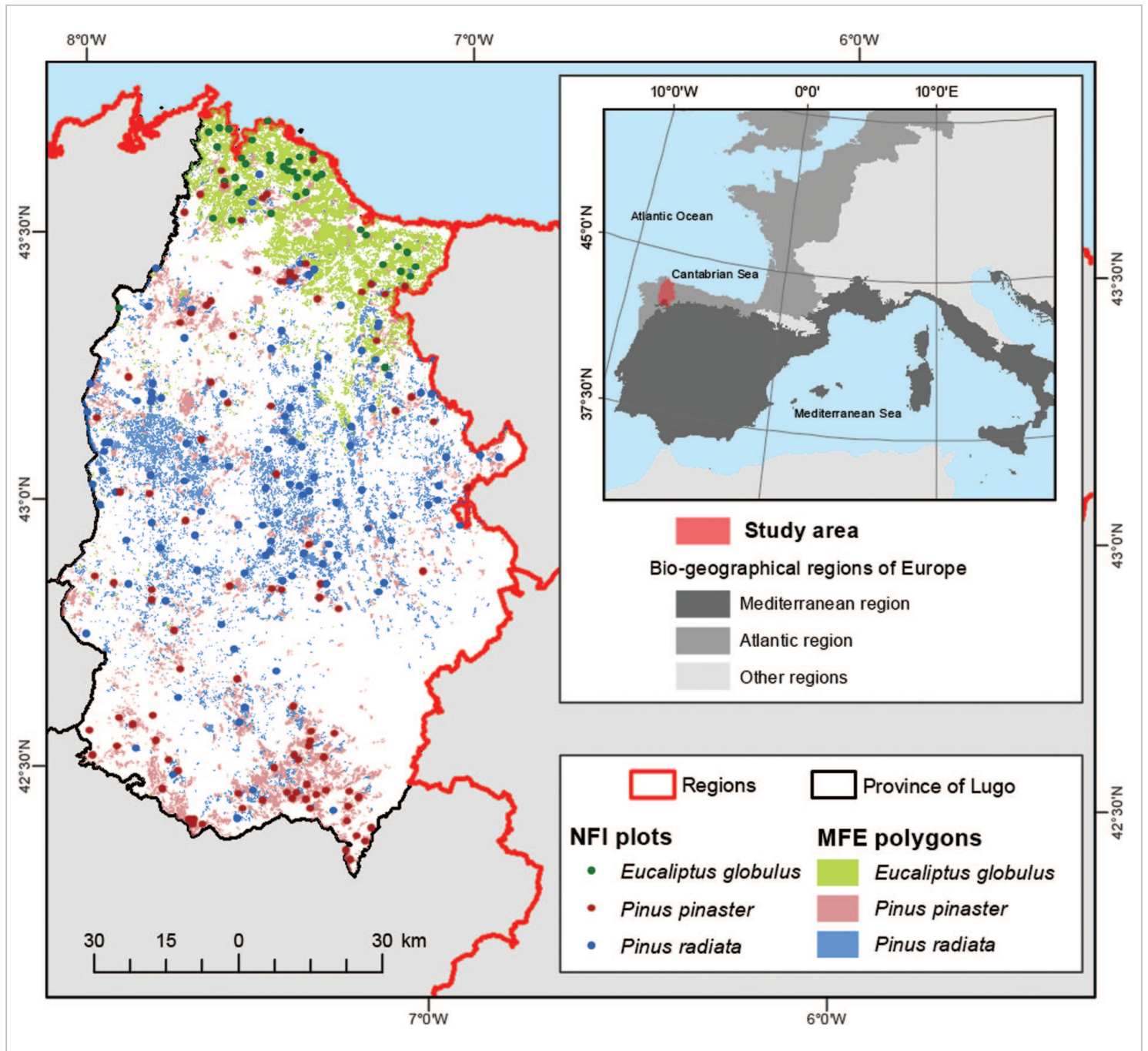

Figura 1. Localización del área de estudio, las parcelas de IFN-4 y los polígonos del IFN-4 según especie mayoritaria. 


\subsection{Material: Datos geográficos y alfa-numéricos}

Los datos utilizados son las parcelas del IFN-4, como datos de campo, y los datos PNOA-LiDAR de $1^{\mathrm{a}}$ y $2^{\mathrm{a}}$ cobertura, como datos LiDAR, correspondientes con la extensión de la provincia de Lugo.

\subsection{Métodos}

\subsubsection{Pre-procesado de datos PNOA-LiDAR}

El pre-procesado de datos se basa en la creación de unidades básicas de trabajo. La unidad básica de trabajo está delimitada por las cuadriculas de la Base topográfica de Galicia a escala 1:10000 (BTG10) con un buffer de 150 metros. Una vez establecidos los límites de cada una de las unidades de trabajo se cruzan con los datos PNOA-LiDAR, de forma que se genera un archivo *.las por cada una de las delimitaciones y cobertura PNOA-LiDAR.

El pre-procesado de los datos PNOA-LiDAR sigue el esquema expuesto en la Fig. 2.

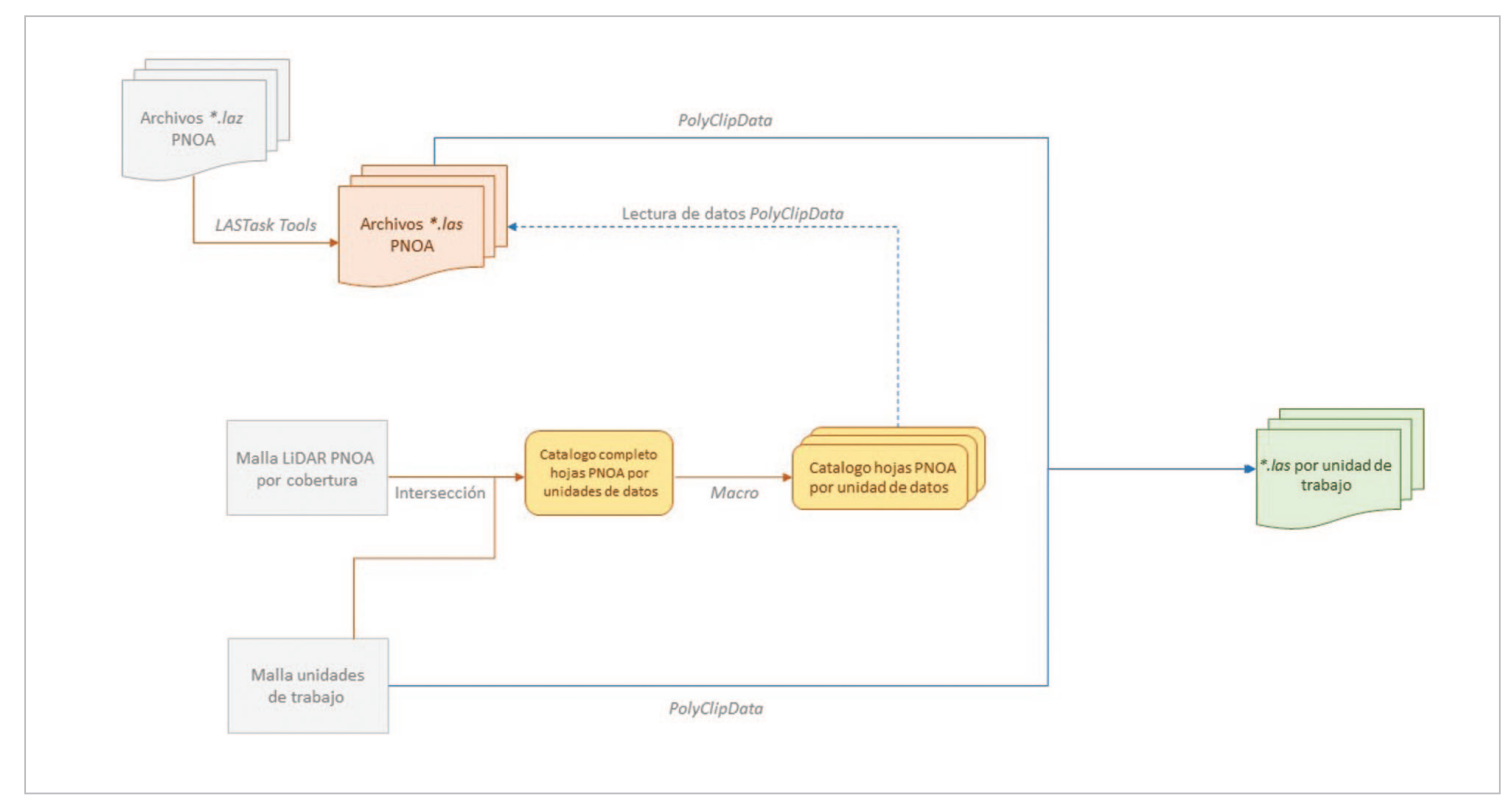

Figura 2. Flujo de trabajo del pre-procesado de los datos por cada cobertura PNOA-LiDAR

Fuente: elaboración propia.

\subsubsection{Procesado de datos PNOA-LiDAR}

Mediante el procesado de los datos PNOA-LiDAR se calculan los variables forestales estructurales, a partir de una superficie de referencia, o MDT, definido previamente. En este caso el MDT de 5 metros del Instituto Geográfico Nacional (IGN) calculado a partir de los datos PNOA-LiDAR de $1^{\text {a }}$ cobertura. 
Las variables forestales estructurales se calculan a partir de las métricas de elevación y de cubierta de dosel (Tab. 1), que se calculan previamente con el software FUSION y ejecutado de forma masiva con lenguaje de programación Python.

Tabla 1. Resumen de las métricas PNOA-LiDAR extraídas para cada parcela.

\begin{tabular}{|c|c|c|c|}
\hline \multicolumn{3}{|c|}{ Métricas ALS } & \multirow{2}{*}{$\begin{array}{c}\text { Descripción } \\
\text { Media }\end{array}$} \\
\hline & $\begin{array}{l}\text { Métricas que expresan } \\
\text { la tendencia central en la }\end{array}$ & hmean & \\
\hline & distribución de alturas ALS & hmode & Moda \\
\hline \multirow{10}{*}{ 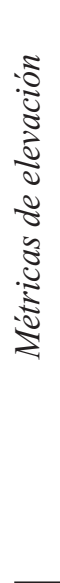 } & \multirow{6}{*}{$\begin{array}{c}\text { Métricas que expresan } \\
\text { la dispersión de la distribución } \\
\text { de alturas ALS }\end{array}$} & $h S D$ & Desviación estándar \\
\hline & & hVAR & Varianza \\
\hline & & $h A A D$ & Desviación media absoluta \\
\hline & & $h I Q$ & Rango intercuartílico \\
\hline & & $h C V$ & Coeficiente de variación \\
\hline & & hmax/hmin & Máximo y mínimo \\
\hline & \multirow{3}{*}{$\begin{array}{c}\text { Métricas que expresan } \\
\text { la forma de la distribución } \\
\text { de altura ALS }\end{array}$} & $h S K w$ & Oblicuidad \\
\hline & & hKurt & Kurtosis \\
\hline & & $C R R$ & Continuidad vertical \\
\hline & $\begin{array}{c}\text { Percentiles de la distribución } \\
\text { de altura ALS }\end{array}$ & $h 01, h 20, \ldots h 95, h 99$ & $\begin{array}{c}\text { 1th,5th, 10th, 20th, 25th, 30th, 40th, } \\
\text { 50th, 60th, 70th, 75th, 80th, 90th, 95th, } \\
\text { 99th percentiles }\end{array}$ \\
\hline \multirow{9}{*}{ 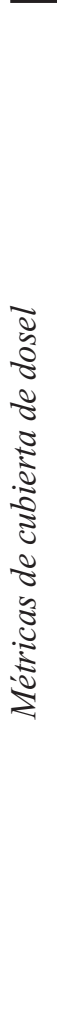 } & \multirow{3}{*}{$\begin{array}{c}\text { Umbral fijo } \\
\text { de ruptura de altura } \\
(\text { HTB })\end{array}$} & $C C$ & $\begin{array}{l}\text { Porcentaje de primeros retornos por } \\
\text { encima de } 2.00 \mathrm{~m} / \text { total de retornos }\end{array}$ \\
\hline & & PARA2 & $\begin{array}{l}\text { Porcentaje de todos los retornos por } \\
\text { encima de } 2.00 \mathrm{~m} / \text { total de retornos }\end{array}$ \\
\hline & & $A R A 2 / T F R$ & $\begin{array}{c}\text { Ratio entre todos los retornos por } \\
\text { encima de } 2.00 \mathrm{~m} \text { y el total de primeros } \\
\text { retornos }\end{array}$ \\
\hline & \multirow{6}{*}{ Variable $H B T$} & PFRAM & $\begin{array}{l}\text { \% de primeros retornos por encima de } \\
\text { la media/total de todos los retornos }\end{array}$ \\
\hline & & PARAM & $\begin{array}{l}\% \text { de todos los retornos por encima de } \\
\text { la media/total de todos los retornos }\end{array}$ \\
\hline & & PARAMO & $\begin{array}{l}\% \text { de todos los retornos por encima de } \\
\text { la modal total de todos los retornos }\end{array}$ \\
\hline & & PFRAMO & $\begin{array}{l}\% \text { de primeros retornos por encima de } \\
\text { la modal total de todos los retornos }\end{array}$ \\
\hline & & ARAM/TFR & $\begin{array}{c}\text { Ratio entre todos los retornos por } \\
\text { encima de la media y el total de } \\
\text { primeros retornos }\end{array}$ \\
\hline & & $A R A M O / T F R$ & $\begin{array}{c}\text { Ratio entre todos los retornos por } \\
\text { encima de la moda y el total } \\
\text { de primeros retornos }\end{array}$ \\
\hline
\end{tabular}

Fuente: elaboración propia. 


\subsubsection{Ajuste de modelos predictivos de VCC e IAVC}

Esta parte del estudio sigue tres partes diferenciadas.

1. Armonización de los datos del IFN-4.

2. Cálculo de los modelos predictivos para las variables de rodal correspondientes al VCC e IAVC.

3. Validación de la estimación del volumen con corteza correspondiente a la $2^{\mathrm{a}}$ cobertura PNOA-LiDAR con respecto a la estimación del volumen con corteza de la $1^{\mathrm{a}}$ cobertura PNOA-LiDAR y su proyección temporal a la $2^{\mathrm{a}}$ cobertura PNOA-LIDAR mediante el modelo IAVC. A continuación, se muestra la metodología de cada uno de los pasos.

\section{Armonización de los datos IFN-4}

Los datos del IFN-4 y los datos PNOA-LIDAR para la $1^{\mathrm{a}}$ cobertura tienen unas discordancias que se corrigen para la correcta realización del estudio. Las armonizaciones que realizan son:

1. Temporal, para ajustar la fecha de captura de datos PNOA-LiDAR y datos IFN-4.

2. Geoespacial, para la determinación de los datos de campo correctamente geoposicionados, determinados a partir de la métrica LiDAR percentil 95 y altura dominante IFN-4.

3. De perturbaciones, para la eliminación de parcelas que vieron alteradas sus dinámicas forestales, y calculada a partir de las métricas LiDAR percentil 95 y fracción de cabida cubierta (FCC) y los valores de altura dominante y FCC del IFN-4.

\section{Ajuste de modelos predictivos VCC e IAVC}

El método utilizado de ajuste de los datos es RF, siendo este un método de clasificación y regresión no paramétrica ampliamente utilizado, que consiste en un conjunto de árboles de decisión. El existo de este método se base en el uso de múltiples árboles desarrollados con diferentes variables independientes que se seleccionan al azar del conjunto original completo de variables. Se utiliza el algoritmo Wrapper (Zhiwei and Xingua, 2010) para la selección de una submuestra óptima de variables que mejor comportamiento presentan.

Los parámetros para cada técnica de regresión son variables. Se utiliza el método CVParameter y seleccionando los parámetros mediante validación cruzada con el clasificador Random Forest y el método $k$-fold. 


\section{Estimación VCC para las coberturas PNOA-LiDAR}

Una vez se obtienen los modelos predictivos para cada una de las especies forestales estudiadas se procede al cálculo del volumen en 2016. Para ello se realizan dos cálculos diferentes:

1. Estimación del VCC de las parcelas del IFN-4 para la primera cobertura PNOA-LiDAR (2009-2010) y posterior proyección temporal de los valores de VCC mediante el modelo de IAVC al instante temporal de la segunda cobertura PNOA-LiDAR (2016).

2. Un cálculo directo de VCC para la segunda cobertura PNOA-LiDAR(2016) a partir del modelo generado previamente. A continuación, se muestra el proceso para cada una de las vías

\section{Resultados}

\subsection{Pre-procesado y procesado de dato PNOA-LiDAR}

Se obtienen 278 archivos *.las, identificados según las hojas BTG10. A partir de estos archivos se calcula el modelo de altura de vegetación (MDHV) para las dos

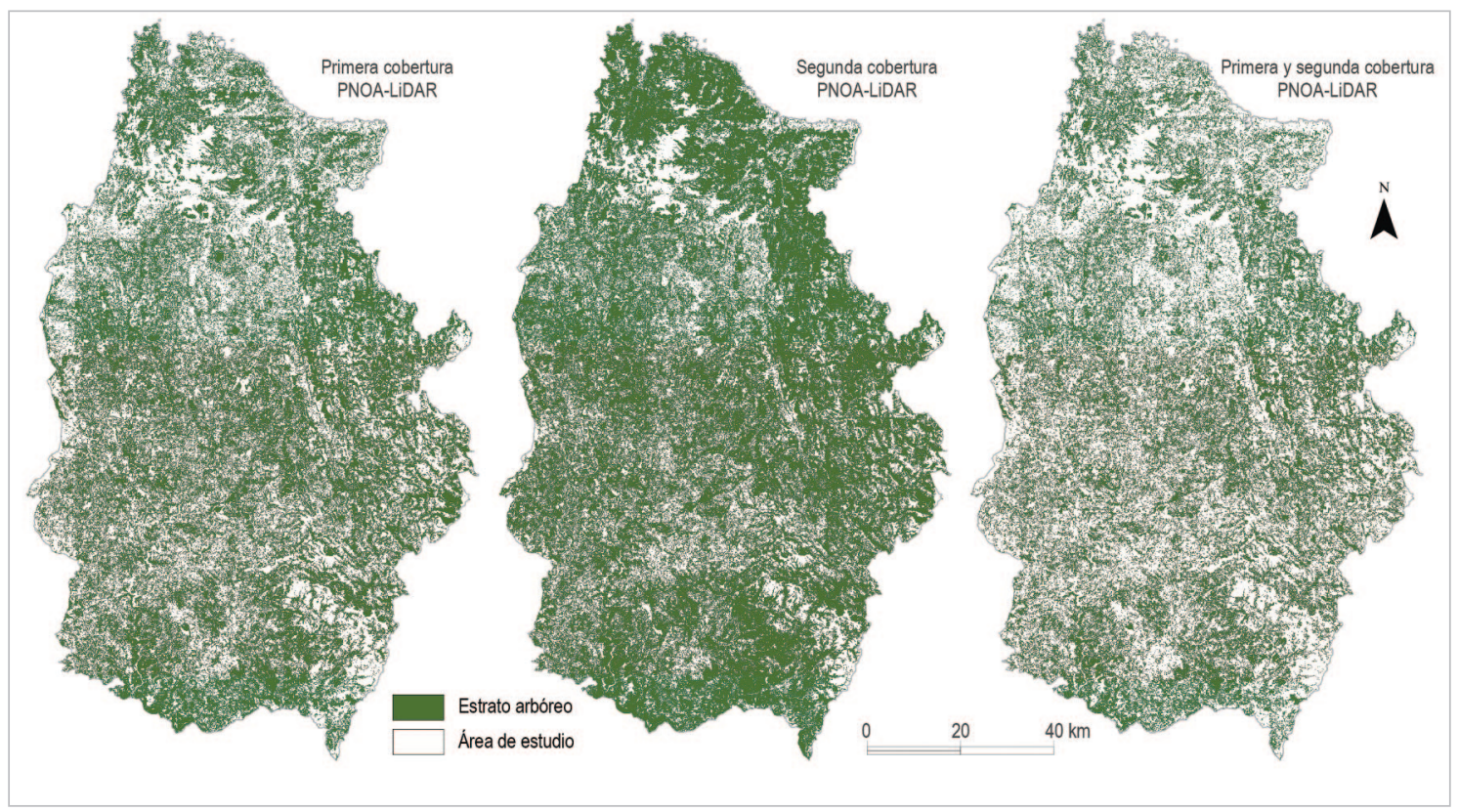

Figura 3. Áreas de la provincia de Lugo donde existe estrato arbóreo (2-70 metros).

Calculado a partir del MDHV obtenido de los datos PNOA-LiDAR de $1^{\mathrm{a}}$ y $2^{\mathrm{a}}$ cobertura. Izquierda, $1^{\mathrm{a}}$ cobertura PNOA-LiDAR; Central, $2^{\mathrm{a}}$ cobertura PNOA-LiDAR; Derecha, zonas con estrato arbóreo en la $1^{\mathrm{a}}$ y $2^{\mathrm{a}}$ cobertura PNOA-LiDAR. 
coberturas PNOA-LiDAR, viendo un aumento del estrato forestal entre 2009 y 2016 y el dinamismo del estrato arbóreo, con un claro aumento de este entre 2009 y 2016 (Fig. 3).

A partir de los datos *.las se obtienen un conjunto de 36 métricas (Tabla 1) de los datos PNOA-LiDAR por cada parcela del IFN-4, a partir de las que se realiza la modelización de VCC e IAVC.

\subsection{Armonización de los datos}

El resultado del proceso de armonización muestra las parcelas aptas para llevar a cabo la modelización y estimación o cálculo del volumen total con corteza.

En la Tab. 2 se muestra el número de parcelas resultantes de cada fase de armonización de datos.

Tabla 2. Número de parcelas por especie del IFN-4 en cada armonización.

\begin{tabular}{cccccc}
\hline Especie & Inicial & A. Temporal & A. Espacial & A. Perturbaciones & A.Coberturas \\
E. globulus & 407 & 407 & 122 & 39 & 25 \\
P.pinaster & 276 & 276 & 138 & 98 & 80 \\
P.radiata & 342 & 342 & 165 & 108 & 83 \\
Total & $\mathbf{1 0 2 5}$ & $\mathbf{1 0 2 5}$ & $\mathbf{4 2 5}$ & $\mathbf{2 4 5}$ & $\mathbf{1 8 8}$ \\
\hline
\end{tabular}

Fuente: elaboración propia.

La armonización espacial muestra un problema de posicionamiento en las parcelas del IFN-4. El proceso de armonización de perturbaciones elimina aquellas muestras que han sufrido una alteración significativa en su estructura vegetal, observando que todas las especies tienen un número elevado de parcelas que sufren alteraciones estructurales. La armonización de coberturas elimina aquellas parcelas del IFN-4 que no han seguido un crecimiento natural, viéndose reducido el tamaño de muestra para todas las especies, especialmente E.globulus.

\subsection{Ajuste de modelos predictivos VCC e IAVC}

Las métricas más representativas, tanto para el modelo VCC como para IAVC, son las asociadas a la altura (Fig. 4) para todas las especies estudiadas.

\subsection{Estimación de volúmenes con corteza para la primera} y segunda cobertura PNOA-LiDAR

\subsubsection{Estimación VCC a partir de la $1^{\mathrm{a}}$ cobertura PNOA-LiDAR y su proyección temporal a la segunda cobertura.}

El modelo lineal ajustado al diagrama de dispersión no reveló ningún problema importante relacionado con la heteroscedasticidad o la falta de normalidad, aunque 


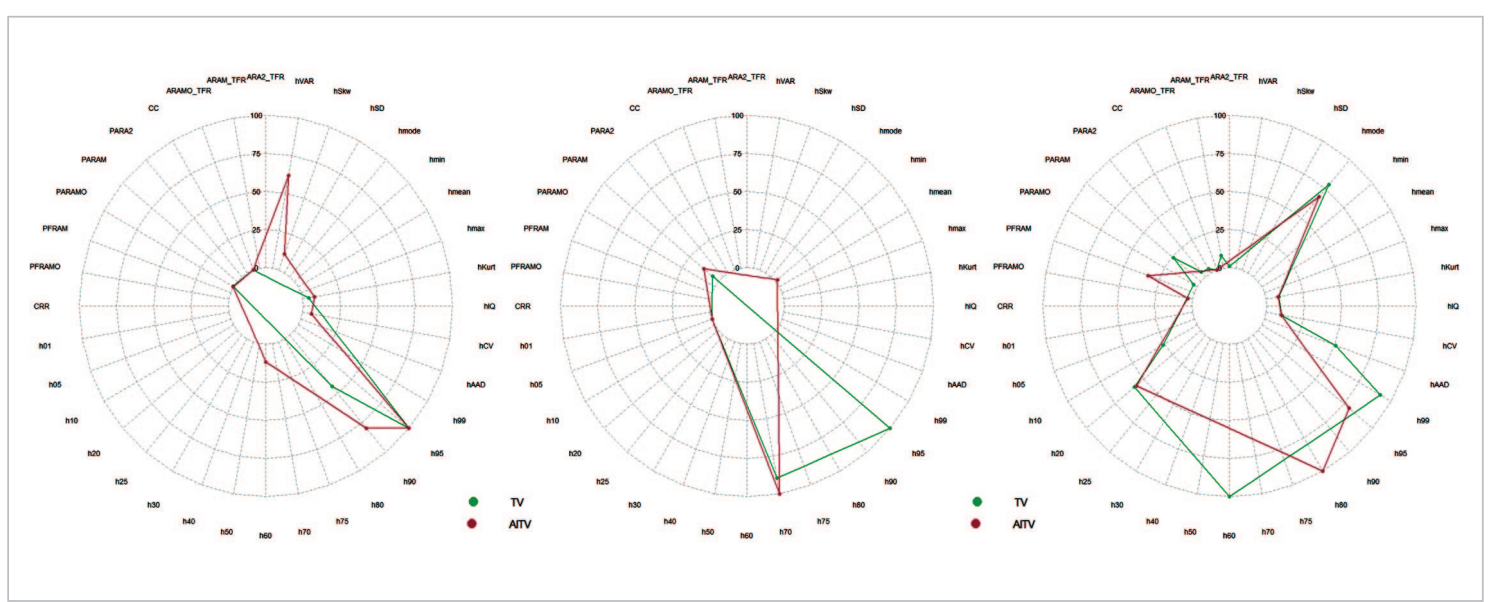

Figura 4. Variables incluidas en el modelo (VIM), para las mejores técnicas, incluido el tipo y la inportacia por variable para cada una de las especies analizadas. E. globulus (izquierda), P. pinaster (centro) y $P$. radiata (derecha). Para garantizar que los valores de importancia variable se expresen en escalas comparables para cada una de las variables de respuesta, las puntuaciones de todos los predictores seleccionados se normalizaron de manera que agregaron un valor unitario (importancia normalizada) o se expresaron como valores relativos: Relativo importancia $=($ VIM-VIMmin $) /($ VIMmax-VIMmin $)$.

Fuente: elaboración propia.

parecía haber una ligera tendencia a la subestimación de los valores altos de VCC para P.radiata (Fig. 5 ).

Al igual que sucede en la modelización de VCC, el IAVC para Pradiata muestra una tendencia a la subestimación en las masas más maduras. Por el contrario, parece producirse una ligera subestimación de los valores altos de IAVC para P.pinaster (Fig. 6).

\subsubsection{Estimación directa del VCC a partir de la $2^{\mathrm{a}}$ cobertura PNOA-LiDAR}

El modelo VCC para E. globulus muestra un modelo sesgado, derivado de la subestimación de los valores predichos de la $1^{\text {a }}$ cobertura PNOA-LiDAR. La es-
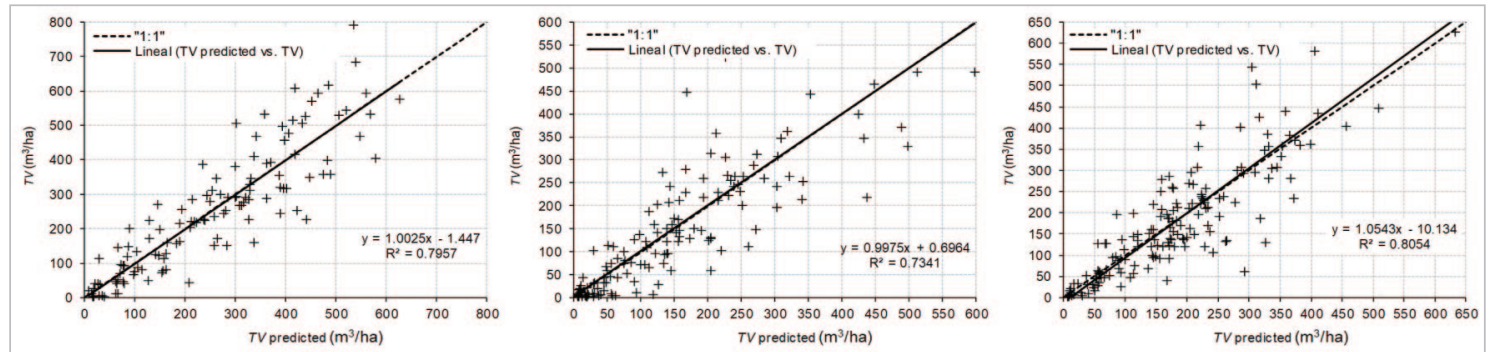

Figura 5. Predicción del nivel de parcela estimado con RF para VCC $\left(\mathrm{m}^{3} \mathrm{ha}^{-1}\right)$

E. globulus (izquierda), P. pinaster (centro) y P. radiata (derecha). La línea continua representa el modelo lineal ajustado a través del diagrama de dispersión de datos y la línea discontinua representa la recta con pendiente igual a 1 . 

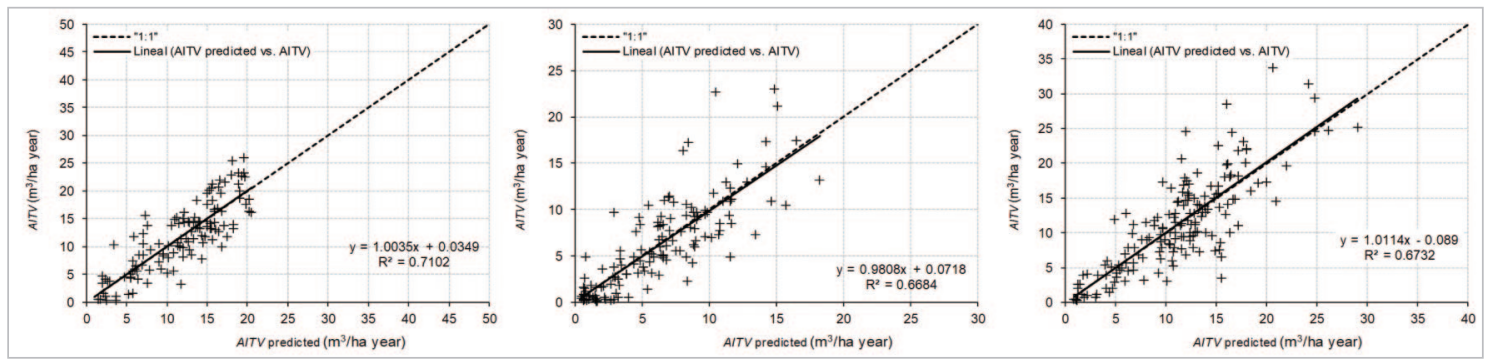

Figura 6. Predicción del nivel de parcela estimado con RF para IAVC ( $\left.\mathrm{m}^{3} \mathrm{ha}^{-1} \mathrm{año}\right)$.

E. globulus (izquierda), $P$. pinaster (centro) y $P$. radiata (derecha). La línea continua representa el modelo lineal ajustado a través del diagrama de dispersión de datos y la línea discontinua representa la recta con pendiente igual a 1 .

Fuente: elaboración propia.

pecie P. pinaster subestima en las masas más jóvenes y sobreestima en las maduras. La estimación de VCC para P.radiata presenta los peores resultados de las tres especies, subestimando los valores de las masas maduras y sobreestimando las jóvenes (Fig. 7).
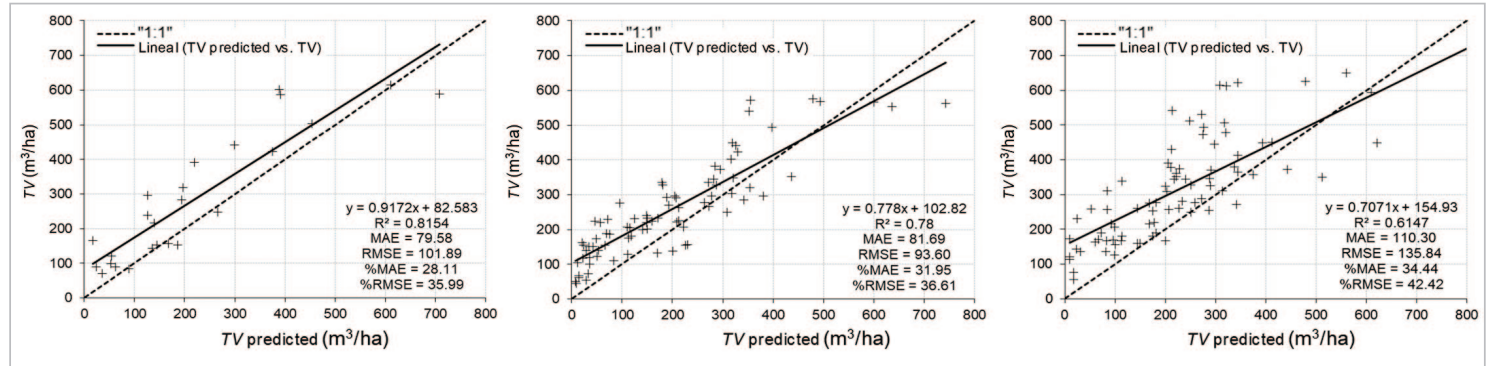

Figura 7. Nivel de parcela predicho frente a los valores observados con RF para VCC $\left(\mathrm{m}^{3} \mathrm{ha}^{-1}\right)$.

E. globulus (izquierda), $P$. pinaster (centro) y $P$. radiata (derecha). La línea continua representa el modelo lineal ajustado a través del diagrama de dispersión de datos y la línea discontinua representa la recta con pendiente igual a 1 .

Fuente: elaboración propia.

\section{Discusión}

A pesar de los desajustes temporales, la no coincidencia temporal entre datos de campo y datos LiDAR, y el problema de geolocalización de los datos, en este trabajo se muestra que es posible integrar los datos PNOA-LiDAR, con datos del IFN4, generando una base de datos de rendimiento de los bosques españoles a través de datos multitemporales PNOA-LIDAR y de proyecciones temporales.

Los resultados de modelización de VCC en 2016 obtuvieron una bondad ajuste en las estadísticas mejores que en estudios anteriores realizados con unos datos similares a los del IFN-4 y la $1^{\text {a }}$ cobertura PNOA-LiDAR. Por ejemplo, los modelos utilizados por Tomé Moran et al. en Murcia obtuvieron valores de rRMSE entre 
47.2 - 44.4\% para IAVC y entre 45.8 - $43.9 \%$ para IAVC para rodales mixtos de pinos, encontrando solo un estudio en la Rioja de Fernández-Landa et al. que se obtienen unos rangos similares, con un R2 de 0.75 y un rango de rRMSE del 26.1$32.3 \%$ para P.sylvestris.

Si se analizan los estudios realizados en la misma área, la mayor parte de los trabajos se realizan con parcelas de investigación y las densidades de los puntos difieren. Por ejemplo, González-Ferreiro et al., en 2012, obtuvo un $\mathrm{R}^{2}=0.69$ y rRMSE del $30 \%$ para densidades iguales a las utilizadas en este estudio, $\mathrm{y} \mathrm{R}^{2}=0.79 \mathrm{y}$ rRMSE del $25 \%$ para densidades de 8 puntos $/ \mathrm{m}^{2}$, en estudios con Pradiata. Gonçalves-Seco et al., en 2011 realizó un estudio para E.globulus en el que obtuvo un valor R2 de 0.81 y una densidad de 4 puntos $/ \mathrm{m} 2$ para el modelo VCC. García-Gutiérrez et al. en 2014 consigue valores $\mathrm{R}^{2}$ entre $0.66-0.70$ y 0.64-0.79 para modelos IAVC ajustados a conjuntos de datos LiDAR de 0.5 a 8 puntos $/ \mathrm{m}^{2}$ respectivamente para Pradiata y E.globulus. A escala local, Navarro et al. (2018). realiza un estudio en Madrid con una densidad de 2.96 puntos $/ \mathrm{m}^{2}$ en parcelas de investigación de P.pinaster, consiguiendo un valor de $\mathrm{R}^{2}=0.79$ y $\mathrm{rRMSE}=25.6 \%$ para el modelo VCC.

\section{Conclusiones}

Este trabajo presenta un procedimiento de armonización de los datos del IFN-4 y los datos LiDAR de las coberturas del PNOA y la definición de una técnica de regresión RF de aprendizaje automático y métodos de base MLR para estimaciones de rendimiento de las especies E.globulus, P.pinaster y $P$. radiata en la provincia de Lugo en 2016.

Además, se muestra una metodología de cálculo de VCC con datos LiDAR multitemporales, aportando resultados validados con los datos obtenidos a través de la proyección del valor IAVC al mismo punto temporal, obteniendo valores válidos, aunque mejorables.

Los datos utilizados, parcelas del IFN-4 presentan problemas de geolocalización, que junto con el desajuste temporal con respecto a los datos PNOA-LiDAR provoca una baja calidad de los mismos, que puede desencadenar en resultados inexactos o incluso no poder realizar el estudios.

En este trabajo se pone de manifiesto que el uso de LiDAR multitemporal hace posible la estimación de los crecimientos y producción de las masas productivas de las especies estudiadas para la provincia de Lugo, siendo su resultado de gran interés en la planificación y economía forestal.

Por otro lado, se ve que los valores obtenidos mediante datos PNOA-LIDAR multitemporales aportan unos resultados similares y válidos a los obtenidos mediante modelización. 


\section{Bibliografía}

Alberdi, I.; Sandoval, V.; Condes, S.; Cañellas, I.; Vallejo, R.; 2016. El Inventario Forestal Nacional español, una herramienta para el conocimiento, la gestión y la conservación de los ecosistemas forestales arbolados. Revista Ecosistemas, 25(3), 88-97. https://doi.org/ 10.1016/j.rse.2019.02.018

Bollandsås, O.M.; Gregoire, T.G.; Næsset, E.; Øyen, B.H.; 2013. Detection of biomass change in a Norwegian mountain forest area using small footprint airborne laser scanner data. Statistical Methods \& Applications, 22(1), 113-129. https://doi.org/10.1007/s10260-012-0220-5

Bollandsås, O.M.; Ene, L.T.; Gobakken, T.; Næsset, E.; 2017. Estimation of biomass change in montane forests in Norway along a $1200 \mathrm{~km}$ latitudinal gradient using airborne laser scanning: a comparison of direct and indirect prediction of change under a model-based inferential approach. Scandinavian journal of forest research, 33(2), 155-165. https://doi.org/ $10.1080 / 02827581.2017 .1338354$

Cao, L.; Coops, N.C.; Innes, J.L.; Sheppard, S.R.; Fu, L.; Ruan, H.; She, G.; 2016. Estimation of forest biomass dynamics in subtropical forests using multi-temporal airborne LiDAR data. Remote Sensing of Environment, 178, 158-171. https://doi.org/10.1016/j.rse.2016. 03.012

Dalponte, M.; Jucker, T.; Liu, S.; Frizzera, L.; Gianelle, D.; 2019. Characterizing forest carbon dynamics using multi-temporal lidar data. Remote Sensing of Environment, 224, 412-420. https://doi.org/10.1016/j.rse.2019.02.018

Englhart, S.; Jubanski, J.; Siegert, F.; 2013. Quantifying dynamics in tropical peat swamp forest biomass with multi-temporal LiDAR datasets. Remote Sensing, 5(5), 2368-2388. https:// doi.org/10.3390/rs5052368

Fernández-Landa, A.; Fernández-Moya, J.; Tomé, J.L.; Algeet-Abarquero, N.; Guillén-Climent, M.L.; Vallejo, R.; Marchamalo, M.; 2018. High resolution forest inventory of pure and mixed stands at regional level combining National Forest Inventory field plots, Landsat, and low density lidar. International journal of remote sensing, 39(14), 4830-4844. https://doi. org/10.1080/01431161.2018.1430406

Garcia-Gutierrez, J.; Gonzalez-Ferreiro, E.; Riquelme-Santos, J.C.; Miranda, D.; Dieguez-Aranda, U.; Navarro-Cerrillo, R.M.; 2014. Evolutionary feature selection to estimate forest stand variables using LiDAR. International Journal of Applied Earth Observation and Geoinformation, 26, 119-131. https://doi.org/10.1016/j.jag.2013.06.005

Gonçalves-Seco, L.; González-Ferreiro, E.; Diéguez-Aranda, U.; Fraga-Bugallo, B.; Crecente, R.; Miranda, D.; 2011. Assessing the attributes of high-density Eucalyptus globulus stands using airborne laser scanner data. International Journal of Remote Sensing, 32(24), 98219841. https://doi.org/10.1080/01431161.2011.593583

González-Ferreiro, E.; Diéguez-Aranda, U.; Miranda, D.; 2012. Estimation of stand variables in Pinus radiata D. Don plantations using different LiDAR pulse densities. Forestry, 85(2), 281292. https://doi.org/10.1093/forestry/cps002

Hopkinson, C.; Chasmer, L.; y Hall, R.J.; 2008. The uncertainty in conifer plantation growth prediction from multi-temporal lidar datasets. Remote Sensing of Environment, 112(3), 11681180. https://doi.org/10.1016/j.rse.2007.07.020

Jiménez, E.; Vega, J.A.; Fernández-Alonso, J.M.; Vega-Nieva, D.; Ortiz, L.; López-Serrano, P.M.; López-Sánchez, C.A.; 2017. Estimation of aboveground forest biomass in Galicia (NW Spain) by the combined use of LiDAR, LANDSAT ETM+ and national forest inventory 
data. iForest-Biogeosciences and Forestry, 10(3), 590. https://doi.org/10.3832/ifor1989-010

Marinelli, D.; Paris, C.; Bruzzone, L.; 2018. A novel approach to 3-D change detection in multitemporal LiDAR data acquired in forest areas. IEEE Transactions on Geoscience and Remote Sensing, 56(6), 3 030-3 046. https://doi.org/10.1109/TGRS.2018.2789660

McRoberts, R.E.; Næsset, E.; Gobakken, T.; Bollandsås, O.M.; 2015. Indirect and direct estimation of forest biomass change using forest inventory and airborne laser scanning data. Remote Sensing of Environment, 164, 36-42. https://doi.org/10.1016/j.rse.2015.02.018

Nilsson, M.; Nordkvist, K.; Jonzén, J.; Lindgren, N.; Axensten, P.; Wallerman, J.; Olsson, H.; 2016. A nationwide forest attribute map of Sweden predicted using airborne laser scanning data and field data from the National Forest Inventory. Remote Sensing of Environment, 194, 447-454. https://doi.org/10.1016/j.rse.2016.10.022

Novo-Fernández, A.; Barrio-Anta, M.A.; Recondo, C.; Cámar-Obregón, A.; López-Sánchez, C.A.; 2019. Integration of National Forest Inventory and Nationwide Airbone Laser Scanning Data to Improve Forest Yield Predictions in North-Western Spain. Remote Sensing. 11(14), 1 693. https://doi.org/10.3390/rs11141693

Réjou-Méchain, M.; Tymen, B.; Blanc, L.; Fauset, S.; Feldpausch, T.R.; Monteagudo, A.; Chave, J.; 2015. Using repeated small-footprint LiDAR acquisitions to infer spatial and temporal variations of a high-biomass Neotropical forest. Remote Sensing of Environment, 169, 93101. https://doi.org/10.1016/j.rse.2015.08.001

Renslow, M.; 2012. Manual of airborne topographic LiDAR. The American Society for Photogrammetry and Remote Sensing, Bethesda, $504 \mathrm{pp}$.

Simonson, W.; Ruiz-Benito, P.; Valladares, F.; Coomes, D.A.; 2016. Modelling above-ground carbon dynamics using multi-temporal airborne lidar: insights from a Mediterranean woodland. Biogeosciences, 13, 961-973. https://doi.org/10.5194/bg-13-961-2016

Tomé-Morán, J.L.; Esteban Cava, J.; Martín Alcón, S.; Escamochero, I.; Fernández-Landa, A.; 2017. ForestMap, Online forest inventories using Murcia Regional Airborne LiDAR Data. XVII Congreso de la Asociación Española de Teledetección. pp. 147-150. Murcia 3-7 octubre 2017.

Wehr, A.; \& Lohr, U.; 1999. Airborne laser scanning — an introduction and overview. ISPRS Journal of photogrammetry and remote sensing, 54(2-3), 68-82. https://doi.org/10.1016 /S0924-2716(99)00011-8

Zhiwei, X.; Xinghua, W.; 2010. Research for information extraction based on wrapper model algorithm. In Proceedings of the Second International Conference on Computer Research and Development, HaiphongCity, Vietnam, 7-10 May 2010; pp. 652-655. https://doi.org/ 10.1109/ICCRD.2010.141

Zhao, K.; Suarez, J.C.; Garcia, M.; Hu, T.; Wang, C.; Londo, A.; 2018. Utility of multitemporal lidar for forest and carbon monitoring: Tree growth, biomass dynamics, and carbon flux. Remote Sensing of Environment, 204, 883-897. https://doi.org/10.1016/j.rse.2017.09.007 